\title{
Identification and characterization of circRNAs as competing endogenous RNAs for miRNA-mRNA in colorectal cancer
}

\author{
Wenliang Yuan ${ }^{1,2,3,4}$, Sihua Peng ${ }^{1,2,3}$, Jingyu Wang ${ }^{5}$, Cai Wei ${ }^{1,2,3}$, Zhen Ye ${ }^{6}$, Ye Wang ${ }^{6}$, Meiliang Wang ${ }^{6}$, Hao Xu $^{6}$, \\ Shouwen Jiang ${ }^{1,2,3}$, Dan Sun ${ }^{1,2,3}$, Chaoxu Dai ${ }^{1,2,3}$, Linhua Jiang ${ }^{4}$, Xiaobo Li ${ }^{\text {Corresp. } 6}$ \\ ${ }^{1}$ Key Laboratory of Exploration and Utilization of Aquatic Genetic Resources, Shanghai Ocean University, Shanghai, China \\ 2 National Pathogen Collection Center for Aquatic Animals, Ministry of Agriculture of China, Shanghai, China \\ 3 International Research Center for Marine Biosciences at Shanghai Ocean University, Ministry of Science and Technology, Shanghai, China \\ 4 School of Optical-Electric and Computer Engineering, University of Shanghai for Science and Technology, Shanghai, China \\ 5 Department of Pathology, The First Affiliated Hospital of Jiaxing University, Jiaxing, China \\ ${ }^{6}$ College of Engineering, Lishui University, Lishui, China \\ Corresponding Author: Xiaobo Li \\ Email address: oboaixil@126.com
}

Background: Recent studies showed that circRNAs are involved in the biological process of some human cancers. However, little is known about their functions in colorectal cancer (CRC).

Methods: Here we first revealed the expression profiles of circRNAs in the CRC tissues and the adjacent non-tumorous tissues using high-throughput sequencing. The sequence feature, chromosome location, alternative splicing and other characteristics of the circRNAs were also explored. The miRNA and mRNA expression profiles were then obtained by analyzing relevant CRC data retrived from the TCGA database. We obtained and analyzed the competing endogenous RNA (ceRNA) network of the top 3 pairs of the largest up-regulated and down-regulated circRNAs.

Results: In this study, we obtained 50,410 circRNAs in the CRC tissue and the adjacent non-tumor tissues, of which $33.7 \%(16,975)$ were new, and revealed differential changes in circRNA expression during colorectal carcinogenesis. We have identified six potential key circRNAs (circPIEZO1-3, hsa_circ_0067163, hsa_circ_0140188, hsa_circ_0002632, hsa_circ_0001998 and hsa_circ_0023990) associated with CRC, which play important roles in carcinogenesis as ceRNA for regulation of miRNAmRNA network. In the subsequent KEGG analysis, several CRC-related pathways were found.

Conclusions: Our findings advance the understanding of the pathogenesis of CRC from the perspective of circRNAs and provide some circRNAs as candidate diagnostic biomarkers or potential therapeutic targets. 
2 Identification and characterization of circRNAs as 3 competing endogenous RNAs for miRNA-mRNA in 4 colorectal cancer

6 Wenliang Yuan ${ }^{1,2,3,4 \ddagger}$, Sihua Peng ${ }^{1,2,3 \ddagger}$, Jingyu Wang ${ }^{5 \ddagger}$, Cai Wei ${ }^{1,2,3}$, Zhen $\mathrm{Ye}^{6}$, Ye Wang ${ }^{6}$, 7 Meiliang Wang ${ }^{6}$, Hao $\mathrm{Xu}^{6}$, Shouwen Jiang ${ }^{1,2,3}$, Dan Sun ${ }^{1,2,3}$, Chaoxu Dai ${ }^{1,2,3}$, Linhua Jiang ${ }^{4}$, and 8 Xiaobo $\mathrm{Li}^{6 *}$

1 Key Laboratory of Exploration and Utilization of Aquatic Genetic Resources (Shanghai Ocean University), Ministry of Education, Shanghai 201306, China

${ }^{2}$ National Pathogen Collection Center for Aquatic Animals, Ministry of Agriculture, Shanghai 201306, China Science and Technology, Shanghai 201306, China

${ }^{4}$ School of Optical-Electric and Computer Engineering, University of Shanghai for Science and Technology, Shanghai 200093, China

$18{ }^{5}$ Department of Pathology, The First Hospital of Jiaxing/The First Affiliated Hospital of Jiaxing 19 University, Jiaxing 314001, China

$20{ }^{6}$ College of Engineering, Lishui University, Lishui 323000, China

$\ddagger$ These authors contribute equally to this work.

* Corresponding authors:

24 Xiaobo Li, PhD, Professor; Email: oboaixil@126.com

\section{Abstract}

Background: Recent studies showed that circRNAs are involved in the biological process of some human cancers. However, little is known about their functions in colorectal cancer (CRC).

Methods: Here we first revealed the expression profiles of circRNAs in the CRC tissues and the adjacent non-tumorous tissues using high-throughput sequencing. The sequence feature, chromosome location, alternative splicing and other characteristics of the circRNAs were also explored. The miRNA and mRNA expression profiles were then obtained by analyzing relevant CRC data retrieved from the TCGA database. We obtained and analyzed the competing endogenous RNA (ceRNA) network of the top three pairs of the largest up-regulated and down-regulated circRNAs. 
35 Results: In this study, we obtained 50,410 circRNAs in the CRC tissue and the adjacent non-tumor tissues, of which $33.7 \%(16,975)$ were new, and revealed differential changes in circRNA expression during colorectal carcinogenesis. We have identified six potential key circRNAs (circPIEZO1-3, hsa_circ_0067163, hsa_circ_0140188, hsa_circ_0002632, hsa_circ_0001998 and hsa_circ_0023990) associated with $\mathrm{CRC}$, which play important roles in carcinogenesis as ceRNA for regulation of miRNAmRNA network. In the subsequent KEGG analysis, several CRC-related pathways were found.

Conclusions: Our findings advance the understanding of the pathogenesis of CRC and provide some circRNAs as candidate diagnostic biomarkers or potential therapeutic targets.

43

\section{Introduction}

Colorectal cancer (CRC) is a common malignant tumor of the digestive system in the world (1.4 million in 2012) [1], and more than 50\% of the patients eventually die from this disease. Chemotherapy is still an indispensable treatment for CRC [2], however, with the advance of molecular biology and cell biology, targeted therapy has become a hotspot in cancer chemotherapy.

Circular RNAs (circRNAs) are a class of non-coding RNAs featuring stable structure, often showing tissue/developmental-phase specific expression [3]. Compared with other non-coding RNA molecules, such as miRNAs and lncRNAs, circRNAs have more desirable biomarker features, such as the stable circular structure, that can be used for disease diagnosis, for example atherosclerosis [4] and gastric cancer [5]. In CRC research, two recent studies demonstrated that circRNA_001569 and circular BANP modulate cell proliferation in colorectal cancer [6, 7]. Recently, it was reported that hsa_circ_0020397 regulates $\mathrm{CRC}$ cell viability, apoptosis, and invasion [8]. Hsiao and colleagues also reported that circular RNA CCDC66 promotes colon cancer growth and metastasis [9].

In this study, we obtained the circRNA expression profiles of the CRC tissues and adjacent non-

58

59

60

61

62

63

64

65

66

67

tumor tissues by high-throughput sequencing, and identified a small number of circRNAs with differential expression; then we analyzed the miRNA and mRNA data for CRC downloaded from the TCGA database; finally, we selected six circRNAs with the most significant differential expressions to analyze their circRNA-miRNA-mRNA network. In addition, Kyoto Gene and Genomic Encyclopedia (KEGG) analyses were performed.

\section{Materials \& Methods}

\section{Patients information}

The CRC tissue specimens and the paired normal mucosa for circRNA detection were available from three CRC patients (two males and one female aged 58-66 years, mean age \pm standard deviation 
68

69

70

71

72

73

74

75

76

77

78

79

80

81

82

83

84

85

86

87

88

89

90

91

92

93

94

95

96

97 98

(SD) $61.3 \pm 4.2$ years) who underwent surgery between May and October 2015 at the First Hospital of Jiaxing, China. The First Hospital of Jiaxing (Jiaxing, Zhejiang, China) granted Ethical approval to carry out the study within its facilities (Ethical Application Ref: FCFHJ-2017023). All the tissues were frozen in liquid nitrogen immediately after the surgery and then stored at $-80^{\circ} \mathrm{C}$ until RNA extraction. All cases were newly diagnosed, histologically confirmed colorectal cancer patients, and had not received any chemotherapy or radiotherapy prior to recruitment.

\section{RNA Sample quality testing}

We used $1 \%$ agarose gel electrophoresis to analyze the purity and integrity of the RNA. The RNA integrity number (RIN) was measured using Agilent RNA 6000 Pico Reagents (Agilent, CA, USA) to assess the RNA quality. Sequencing was performed if the samples RIN values were greater than eight. The Qubit 2.0 instrument was used to accurately measure the RNA concentration.

\section{Sequencing Library Preparation and circRNA Sequencing}

A total amount of $1.5 \mu \mathrm{g}$ RNA per sample was used as input material for the RNA sample preparations. Sequencing libraries were generated using NEBNext ${ }^{\circledR}$ Ultra $^{\text {TM }}$ RNA Library Prep Kit for Illumina ${ }^{\circledR}$ (NEB,USA) following manufacturer's instructions. Then $3 \mu$ l USER Enzyme (NEB, USA) was used with size-selected, adaptor-ligated cDNA at $37^{\circ} \mathrm{C}$ for 15 min followed by 5 min at $95^{\circ} \mathrm{C}$ before PCR. Then PCR was performed with Phusion High-Fidelity DNA polymerase, Universal PCR primers and Index (X) Primer. Finally, products were purified (AMPure XP system) and library quality was assessed on the Agilent Bioanalyzer 2100 system.

After cluster generation, the prepared libraries were sequenced on an Illumina Hiseq 4000 platform and 150 bp paired-end reads were generated.

\section{Screening and identification of colorectal cancer-associated circRNAs}

To identify the circRNAs in the RNA-Seq data, the sequence reads were firstly mapped to the human reference genome (GRCh37/hg19, Feb., 2009) using TopHat2 (v2.1.0) [10]; Then, back-spliced ordering reads were extracted for circRNA prediction using CIRCexplorer [11]. These circRNAs were annotated by searching the circBase database [12] and the deepBase database [13]. Finally, differentially expressed circRNAs were identified using edgeR [14], according to the criteria of a $\left|\log _{2} \mathrm{FC}\right|>1.5$ and Pvalue $<0.05$.

\section{Prediction of the potential coding ability of circRNAs}

It took two steps to predict the potential coding ability of differentially expressed circRNAs through bioinformatics method. Firstly, an online tool getorf (http://emboss.bioinformatics.nl/cgi- 
99

100

101

102

103

104

105

106

107

108

109

110

111

112

113

114

115

116

117

118

119

120

121

122

123

124

125

126

127

128

129

bin/emboss/getorf) was used to determine whether a circRNA has a open reading frame (ORF). Then, we blasted the circRNA sequences against all Internal Ribosome Entry Site (IRES) sequences using IRESite tool [15], and the circRNAs with E Value $<0.05$ were considered to have potential encoding capability.

\section{Identification of differentially expressed miR $N A s$ and $m R N A s$}

To verify that circRNAs function as sponges or inhibitors of their interacting miRNAs, transcriptome profiling datasets were downloaded from TCGA. A data of 41 normal and 480 tumor samples for mRNA analysis were obtained. Similarly, the data of eight normal and 457 tumor samples were obtained for the miRNAs analysis by the same method. Finally, the differentially expressed miRNAs and mRNAs were identified using edgeR, according to the criteria of a fold change $>2.0$ and false discovery rate $($ FDR $)<0.01$.

\section{miRNAs prediction, co-expression network and function analysis}

The putative circRNA/miRNA interactions were investigated by miRanda [16] using the miRNA list from miRBase release 20.0 [17]. The putative target genes of the miRNAs were predicted using the intersection of miRTarBase [18] and miRDB [19]. The information on the circRNAs of interest was obtained by CSCD [20].

The circRNA-miRNA-mRNA interaction network was constructed by Cytoscape. Cytoscape two plugins (ClueGO and CluePedia) were used for KEGG analysis, showing only pathway with P-Value < 0.05 .

\section{Results}

\section{Sequencing data}

The sequencing yielded a total of $79.024 \mathrm{G}$ of Raw data, and the filtered clean data totaled 72.874 G. The quality of the sequencing data was detailed in supplementary file 1 .

\section{General characteristics of circRNAs in CRC}

A total of 50,410 circRNAs derived from 9,620 host genes were identified in the human CRC tissues and the adjacent non-tumorous tissues. Among them, 28,032 were found in circBase, 5,403 were included in deepBase, and remaining 16,975 accounting for $33.7 \%$ of the total circRNAs were observed for the first time in this study.

According to their host gene location, the 50,410 circRNAs were widely distributed on all the chromosomes (Fig. 1A). Specifically, only chromosome 1 and chromosome 2 produced more than 4,000 circRNAs. Most other chromosomes generated more than one thousand circRNAs, except chromosome 
130

131

132

133

134

135

136

137

138

139

140

141

142

143

144

145

146

147

148

149

150

151

152

153

154

155

156

157

158

159

160

161

162

163

164

165

21, Y and chrUn (with 542, 81 and 3 circRNAs, respectively). Our data showed that 49,801 (98.8\%) circRNAs were excluded from the first or last exons of their host genes (Fig. 1B). In addition, we found that about $66.5 \%$ of the host genes produced multiple circRNA isoforms (Fig. 1C). We found that the BIRC6 host gene produced the highest numbers of circRNAs isoforms. Interestingly, it was described in other studies that BIRC6 over-expression is a predictor of poor prognosis in CRC [21]. Most exonic circRNAs consisted of multiple exons, with the most circRNAs containing two or three exons, and the maximum number of exons in a circRNA was 48 (Fig. 1D).

\section{Insert Fig. 1 here.}

Fig. 1 General characteristics of circRNAs in CRC. (A) Genomic features of circRNAs expressed in human CRC. Chromosomal distribution of the circRNAs. (B) Distribution of the back-spliced exons in circRNAs. (C) Distribution of the number of different types of circRNA transcripts from each circRNA host gene. (D) Distribution of the number of back-spliced exons in each circRNA.

\section{Screening of the differentially expressed circRNA}

The differentially expressed circRNAs between the CRC tissues and the adjacent non-tumorous tissues were identified. Finally, 98 circRNAs were identified, of which 49 were up-regulated and 49 were down-regulated (Supplementary file 2). The hierarchical clustering (Fig. 2A) and volcano plots (Fig. 2B) showed the variation of circRNA expression between the normal and the CRC samples. Additionally, the host genes of these differentially expressed circRNAs were derived from exonic regions (94), intronic regions (1, circMYO7B-3) (Fig. 2C), etc.

To predict the potential coding ability of the differentially expressed circRNAs, we found that 69 circRNAs (70\%) contained at least one ORF, but only eight circRNAs had IRESs (Fig. 2D). To investigate the functional association of the host genes of the differentially expressed circRNAs in $\mathrm{CRC}$, we analyzed the genes using the GeneMANIA plugin in the Cytoscape software (Fig. 2E). Most of the network interactions were co-expression, physical interactions and genetic interactions. The complex interaction between host genes suggests that this correlation may also exist between differentially expressed circRNAs.

\section{Insert Fig. 2 here.}

Fig. 2 Differential expression of circRNAs in CRC tissues. (A) Hierarchical clustering analysis of the circRNAs. CRC-D-A, CRC-D-B and CRC-D-C are adjacent normal tissue samples. The remaining three are cancer tissue samples. (B) Volcano plots are constructed using the fold-change values and q-values. The red dots in the figure represent statistically significant differentially expressed circRNAs. (C) Distribution of genomic regions that differentially expressed circRNAs derived from: exonic, intronic regions, etc. (D) Potentially encoded protein analysis of differentially expressed circRNAs. (E) GeneMANIA network of host genes of differentially expressed circRNAs. 
166 Screening of differentially expressed miRNAs and $m R N A s$

167

168

169

170

171

172

173

174

175

176

177

178

179

180

181

182

183

184

185

186

187

188

189

190

191

192

193

194

195

196

197

198

According to the criteria of $|\log 2 \mathrm{FC}|>2$ and q-value $<0.01,245$ pre-miRNAs (DE_pmiRNA) and 2,083 mRNAs (DE_mRNA) were identified as aberrantly expressed in the CRC tissues compared with the adjacent non-tumorous tissues (Supplementary file 3 and Supplementary file 4). It was found that many miRNAs and mRNAs were up-regulated or down-regulated more than 100-fold (Fig. 3A-B).

\section{Insert Fig. 3 here.}

Fig. 3 Differential expression analysis and interaction analysis of miRNAs and mRNAs. Volcano plots showing expression profile of pre-miRNAs (A) and mRNAs (B). (C) The intersection of the differentially expressed pre-miRNAs (DE_pmiRNA) and 1,666 pre-miRNAs. (D) The intersection of the differentially expressed mRNA (DE_mRNA) and 3707 miRNA target genes. (E) Comparison of data sizes before and after data processing. Purple indicates retained data and blue indicates discarded data.

\section{Interaction between differentially expressed circRNAs, miRNAs and mRNAs}

Evidence showed that circRNAs function as sponges or inhibitors of their interacting miRNAs to terminate regulation of their target genes $[8,9]$. We obtained 1,666 pre-miRNAs including binding sites of the differentially expressed circRNAs, and then obtained 3,707 target genes of these pre-miRNAs by searching the three databases. Furthermore, by analyzing DE_pmiRNA and these pre-miRNAs, we obtained 192 miRNAs in the intersection, so these miRNAs can interact with the circRNAs (Fig. 3C). Similarly, we obtained 225 DE_RNAs related to the differentially expressed circRNA (Fig. 3D), and in this process, 40 DE_pmiRNAs were discarded because their target genes did not appear in this set (Fig. 3E). Interestingly, even if only 123-DE_pmiRNAs were retained, all of the differentially expressed circRNAs were still retained.

\section{Networks Regulated by circRNAs}

We selected the top three down-regulated (circPIEZO1-3, hsa_circ_0067163, and hsa_circ_0140188) and up-regulated circRNAs (hsa_circ_0002632, hsa_circ_0001998 and hsa_circ_0023990) as the hub components referring to recent studies [22]. As shown in Fig. 4A, we found that all the six circRNAs belonged to the exonic circRNA and were all cyclized by multiple exons. We also found that the expression of hsa_circ_0140188 was significantly down-regulated, and the expression of its host gene DMD was decreased. Similarly, hsa_circ_0023990 and the host gene NOX4 were highly expressed. However, this consistent change in expression did not occur in the remaining four cirRNAs and their host genes, probably because circRNAs have a higher stability.

To investigate the potential mechanisms of circRNA in the development and progression of CRC, we constructed the circRNA-miRNA-mRNA interaction network for these six circRNAs. The ceRNA 
199

200

201

202

203

204

205

206

207

208

209

210

211

212

213

214

215

216

217

218

219

220

221

222

223

224

225

226

227

228

229

230

231

232

233

interaction network consists of six circRNAs, 35 DE_miRNAs and 64 DE_mRNAs (Fig. 4B). By querying the clinical data in the TCGA database, we found that the expression levels of the six ceRNArelated mRNAs significantly correlated to the survival time of the CRC patients (Fig. 4C), suggesting that circRNAs-selected may have prognostic value. We found that a high expression of hsa_circ_0023990 significantly improved the survival time in the patients with CRC due to the high expressions of SOX1, AQP6 and ITGBL1. Similarly, a low expression of hsa_circ_0067163 correlates to a poor survival due to a low expression of TPM2.

Insert Fig. 4 here.

Fig. 4 Information on six hub circRNAs. (A) The top three up-regulated and down-regulated circRNAs. The outer loop represents the exon that constitutes the circRNA, the innermost green ring represents the ORF, the middle red triangle represents the miRNA response element, and the blue cross point represents the RNA binding protein. (B) CircRNAs-miRNAs-mRNAs network. The red circle denotes the down-regulated circRNAs, the green circle denotes the up-regulated circRNAs, the blue inverted triangle denotes miRNAs, and the purple rectangle denotes the mRNAs. (C) Survival analysis of ceRNA-related mRNA. The red line denotes the high expression of the gene and the blue line denotes the low expression. (D) The KEGG pathway analysis of the top three circRNA pairs in the up-regulation and down-regulation circRNAs.

\section{Functional enrichment analysis of circRNAs}

The functional roles of most circRNAs have not been characterized, however, it would be beneficial to predict signaling pathways involving circRNAs by bioinformatics methods. Therefore, according to the obtained ceRNA network and the target genes of the miRNA in the network, the KEGG pathway analysis of the six circRNAs was performed (Fig. 4D). There were 17 KEGG pathways significantly enriched in our study $(\mathrm{P}<0.01)$. Among these pathways, some were directly linked to cancer pathogenesis, such as colorectal cancer, p53 signaling pathway [23], TGF- $\beta$ signaling pathways [24] and microRNAs in cancer. Interestingly, although other pathways, such as cellular senescence [25] and Foxo signaling pathway [26], seemed not to be directly related to CRC, they were also found associated with the development of multiple diseases.

\section{Discussion}

Up to now, many circRNAs have been found in various human normal or diseased tissues. Researchers have identified 8,045 in heart, 3,982 in liver [27], 15,996 in testis [28] and 65,731 in normal human brain [29]. In our study, we predicted 50,410 circRNAs in the normal and diseased human colorectal tissues. Compared with other organs (for example heart, liver and testis), the expressions of the circRNAs in the human colorectal tissues are the most abundant. Our data showed that most circRNAs are excluded from the first or last exons of their host genes, which is consistent with previous research

PeerJ reviewing PDF | (2019:04:36876:2:0:NEW 25 Jul 2019) 
234 that back-spliced events are generally difficult to occur in the first or last exons of the host genes [30]. In 235 the present study, we found that $66.5 \%$ of the 9,620 host genes produce multiple circRNA isoforms, 236 suggesting that there are other factors contributing to the occurrence of back-spliced events, for example, 237 non-repetitive sequences are largely included in these "hot-spot" genes [25].

238 Research showed that most circRNAs are derived from exonic regions and 5' UTR sequences 239 [29]. Data analysis of our differentially expressed circRNAs also supports this view. Recent studies 240 showed that circRNAs directly translate proteins and participate in various physiological processes [31, $24132]$. We analyzed the differentially expressed circRNAs and found that most of them contained ORF and 242 IRES, indicating that these circRNAs have potential coding ability.

As is known, some oncogenes, such as RNA binding protein, ribosomal protein S5 (RPS5) and 5244 hydroxytryptamine receptor 4 (HTR4), are differentially expressed in CRC compared with adjacent 245 normal tissues [33, 34]. In our differentially expressed circRNA we found, the host genes of 246 hsa_circ_0128314 and hsa_circ_0005598 are HTR4 and RPS5, respectively. Therefore, we believe that 247 some oncogenes will not affect their carcinogenic properties even if they are cyclized during 248 transcription.

CeRNA hypothesis describes the mechanism for a class of RNAs with miRNA binding sites that competitively bind to miRNAs to inhibit their regulation of the target genes $[35,36]$. The carcinogenic mechanism of circRNAs may occur through their miRNA-mediated effects on the gene expression, as circRNAs have more miRNA binding sites and are highly stable [37, 38]. In our study, based on the ceRNA hypothesis, we utilized paired circRNA, miRNA and mRNA expression profiles of the CRC patients combined with experimentally validated miRNA-target interactions to reconstruct circRNAassociated ceRNA network for the progression of CRC. However, Our findings are preliminary and has some limitations because our findings were only based on bioinformatics analyses and extensive wet-lab validation experiments are needed. As for our future experimental validation plan, we will focus on the in vitro validation of differential expression of hsa_circ_0023990 to verify its correlation with differentially expressed multiple mRNAs, such as SOX1, AQP6 and ITGBL1.

In the ceRNA network of the selected "hot-spot" circRNAs, we found that some miRNAs have 261 been confirmed to promote colorectal cancer pathogenesis for their expression difference by other studies, 262 such as hsa-miR-29c-3p [39], suggesting that circRNA plays a role in the development of cancer by 263 absorbing functional miRNAs to regulate the expression of corresponding genes. In addition, we also 264 found some more complex regulatory relationships between circRNAs and miRNAs, for example, a high 265 expression of hsa_circ_0067163 and a low expression of hsa-circ_0001998 simultaneously acted as a 
The occurrence of colorectal cancer is not simply caused by a single signal pathway. Its

268

269

270

271

272

273

274

275

276

277

278

279

280

281

282

283

284

285

286

287

288

289

290

291

298

295

296

297

298

299

300

301

302

303

304

305

306 occurrence and development are the result of the accumulation of multiple signal pathways, which are regulated by the network interlaced downstream of the pathway. Abnormalities in each pathway may cause disorder and/or cause colorectal cancer. The TGF- $\beta$ signaling pathway regulates cell proliferation, differentiation, migration, apoptosis, and regulates stem cell repair [40]. The transcriptional co-activator with PDZ-binding motif and Yes-associated protein integrates with Wnt and TGF- $\beta$ signaling in several cells and may have a significant effect on intestinal cell proliferation, differentiation and other functions [24].

\section{Conclusions}

In summary, in this study, we obtained 50,410 circRNAs in the CRC tissue and the adjacent nontumor tissues, of which $33.7 \%(16,975)$ were new, and revealed differential changes in the circRNA expressions during colorectal carcinogenesis. We have identified six potential key circRNAs associated with CRC, which play important roles in carcinogenesis as ceRNA for the regulation of the miRNAmRNA network. Our findings advance the understanding of the pathogenesis of CRC from the perspective of circRNAs and provide some circRNAs as candidate diagnostic biomarkers or potential therapeutic targets.

\section{Acknowledgements}

We thank Dr. Xiaoning Peng and Dr. Zuozhou Chen for their helpful suggestions.

\section{Additional files}

Additional file1: Summary of the quality of the raw sequencing data

Additional file2: Differentially expressed circRNAs in the CRC

Additional file3: Differentially expressed miRNAs in the CRC

Additional file4: Differentially expressed mRNAs in the CRC

\section{Availability of data and materials}
The RNA-seq data are deposited under NCBI
BioProject (ID: PRJNA521856).

\section{References}

1. McGuire S: World Cancer Report 2014. Geneva, Switzerland: World Health Organization, International Agency for Research on Cancer, WHO Press, 2015. Advances in Nutrition 2016, 7(2):418-419.

2. Gill S: Adjuvant therapy for resected high-risk colon cancer: Current standards and controversies. Indian Journal of Medical and Paediatric Oncology : Official Journal of Indian Society of Medical \& Paediatric Oncology 2014, 35(3):197-202.

3. Memczak S, Jens M, Elefsinioti A, Torti F, Krueger J, Rybak A, Maier L, Mackowiak SD, Gregersen LH, Munschauer M: Circular RNAs are a large class of animal RNAs with regulatory potency. Nature 2013, 495(7441):333. 
307 4. Burd CE, Jeck WR, Liu Y, Sanoff HK, Wang Z, Sharpless NE: Expression of linear and novel circular forms of an INK4/ARF-associated non-coding RNA correlates with atherosclerosis risk. PLoS genetics 2010, 6(12):e1001233.

5. Li P, Chen S, Chen H, Mo X, Li T, Shao Y, Xiao B, Guo J: Using circular RNA as a novel type of biomarker in the screening of gastric cancer. Clin Chim Acta 2015, 444:132-136.

6. Zhu M, Xu Y, Chen Y, Yan F: Circular BANP, an upregulated circular RNA that modulates cell proliferation in colorectal cancer. Biomed Pharmacother 2017, 88:138-144.

7. Xie H, Ren X, Xin S, Lan X, Lu G, Lin Y, Yang S, Zeng Z, Liao W, Ding Y-Q: Emerging roles of circRNA_001569 targeting miR-145 in the proliferation and invasion of colorectal cancer. Oncotarget 2016, 7(18):26680.

8. Zhang Xl, Xu Ll, Wang F: Hsa_circ_0020397 regulates colorectal cancer cell viability, apoptosis and invasion by promoting the expression of the miR - 138 targets TERT and PD - L1. Cell Biol Int 2017, 41(9):1056-1064.

9. Hsiao K-Y, Lin Y-C, Gupta SK, Chang N, Yen L, Sun HS, Tsai S-J: Non-coding effects of circular RNA CD promote colon cancer growth and metastasis. Cancer research 2017:77 (79):2339.

10. Trapnell C, Roberts A, Goff L, Pertea G, Kim D, Kelley DR, Pimentel H, Salzberg SL, Rinn JL, Pachter L: Differential gene and transcript expression analysis of RNA-seq experiments with TopHat and Cufflinks. Nat Protoc 2012, 7(3):562.

11. Zhang XO, Wang HB, Zhang Y, Lu X, Chen LL, Yang L: Complementary sequence-mediated exon circularization. Cell 2014, 159(1):134-147.

12. Glažar P, Papavasileiou P, Rajewsky N: circBase: a database for circular RNAs. Rna 2014, 20(11):16661670.

13. Yang J-H, Shao P, Zhou H, Chen Y-Q, Qu L-H: deepBase: a database for deeply annotating and mining deep sequencing data. Nucleic Acids Res 2009, 38(suppl_1):D123-D130.

14. Robinson MD, McCarthy DJ, Smyth GK: edgeR: a Bioconductor package for differential expression analysis of digital gene expression data. Bioinformatics 2010, 26(1):139-140.

15. Mokrejš M, Mašek T, Vopálenský V, Hlubuček P, Delbos P, Pospíšek M: IRESite-a tool for the examination of viral and cellular internal ribosome entry sites. Nucleic Acids Res 2009, 38(suppl_1):D131-D136.

16. Betel D, Wilson M, Gabow A, Marks DS, Sander C: The microRNA. org resource: targets and expression. Nucleic Acids Res 2008, 36(suppl_1):D149-D153.

17. Griffiths-Jones S, Grocock RJ, Van Dongen S, Bateman A, Enright AJ: miRBase: microRNA sequences, targets and gene nomenclature. Nucleic Acids Res 2006, 34(suppl_1):D140-D144.

18. Friedman RC, Farh KK-H, Burge CB, Bartel DP: Most mammalian mRNAs are conserved targets of microRNAs. Genome research 2009, 19(1):92-105.

19. Wong N, Wang X: miRDB: an online resource for microRNA target prediction and functional annotations. Nucleic Acids Res 2014, 43(D1):D146-D152.

20. Xia S, Feng J, Chen K, Ma Y, Gong J, Cai F, Jin Y, Gao Y, Xia L, Chang H: CSCD: a database for cancer-specific circular RNAs. Nucleic Acids Res 2018, 46(Database issue):D925-D929.

21. Hu T, Weng S, Tang W, Xue R, Chen S, Cai G, Cai Y, Shen X, Zhang S, Dong L: Overexpression of BIRC6 is a predictor of prognosis for colorectal cancer. PLoS One 2015, 10(5):e0125281.

22. Gargouri M, Park J-J, Holguin FO, Kim M-J, Wang H, Deshpande RR, Shachar-Hill Y, Hicks LM, Gang DR: Identification of regulatory network hubs that control lipid metabolism in Chlamydomonas reinhardtii. $J$ Exp Bot 2015, 66(15):4551-4566.

23. Slattery ML, Mullany LE, Wolff RK, Sakoda LC, Samowitz WS, Herrick JS: The p53-signaling pathway and colorectal cancer: Interactions between downstream p53 target genes and miRNAs. Genomics 2018.

24. $\mathrm{Xu} \mathrm{Y,} \mathrm{Pasche} \mathrm{B:} \mathrm{TGF-} \beta$ signaling alterations and susceptibility to colorectal cancer. Human molecular genetics 2007, 16(R1):R14-R20.

25. Schmitt CA: Cellular senescence and cancer treatment. Biochimica et Biophysica Acta (BBA)-Reviews on Cancer 2007, 1775(1):5-20.

26. Liang Z, Wang X, Xu X, Xie B, Ji A, Meng S, Li S, Zhu Y, Wu J, Hu Z: MicroRNA-608 inhibits proliferation of bladder cancer via AKT/FOXO3a signaling pathway. Molecular cancer 2017, 16(1):96.

27. Zheng Q, Bao C, Guo W, Li S, Chen J, Chen B, Luo Y, Lyu D, Li Y, Shi G: Circular RNA profiling reveals an abundant circHIPK3 that regulates cell growth by sponging multiple miRNAs. Nature communications 2016, 7:11215. 
28. Dong W-W, Li H-M, Qing X-R, Huang D-H, Li H-G: Identification and characterization of human testis derived circular RNAs and their existence in seminal plasma. Scientific reports 2016, 6:39080.

29. Rybak-Wolf A, Stottmeister C, Glažar P, Jens M, Pino N, Giusti S, Hanan M, Behm M, Bartok O, AshwalFluss R: Circular RNAs in the mammalian brain are highly abundant, conserved, and dynamically expressed. Molecular cell 2015, 58(5):870-885.

30. Zhang X-O, Wang H-B, Zhang Y, Lu X, Chen L-L, Yang L: Complementary sequence-mediated exon circularization. Cell 2014, 159(1):134-147.

31. Yang Y, Fan X, Mao M, Song X, Wu P, Zhang Y, Jin Y, Yang Y, Chen L-L, Wang Y: Extensive translation of circular RNAs driven by N 6-methyladenosine. Cell research 2017, 27(5):626. Molecular cell 2017, 66(1):22-37. e29.

33. Shimoyama M, Laulederkind SJ, De Pons J, Nigam R, Smith JR, Tutaj M, Petri V, Hayman GT, Wang S-J, Ghiasvand O: Exploring human disease using the Rat Genome Database. Disease models \& mechanisms 2016, 9(10):1089-1095.

34. Hou N, Guo Z, Zhao G, Jia G, Luo B, Shen X, Bai Y: Inhibition of micro RNA - 21 - 3p suppresses proliferation as well as invasion and induces apoptosis by targeting RNA - binding protein with multiple splicing through Smad4/extra cellular signal - regulated protein kinase signalling pathway in human colorectal cancer HCT 116 cells. Clinical and Experimental Pharmacology and Physiology 2018.

35. Tay Y, Rinn J, Pandolfi PP: The multilayered complexity of ceRNA crosstalk and competition. Nature 2014, 505(7483):344.

36. Salmena L, Poliseno L, Tay Y, Kats L, Pandolfi PP: A ceRNA hypothesis: the Rosetta Stone of a hidden RNA language? Cell 2011, 146(3):353-358.

37. Wilusz JE, Sharp PA: A circuitous route to noncoding RNA. Science 2013, 340(6131):440-441.

38. Guo JU, Agarwal V, Guo H, Bartel DP: Expanded identification and characterization of mammalian circular RNAs. Genome biology 2014, 15(7):409.

39. Chen G, Zhou T, Li Y, Yu Z, Sun L: p53 target miR-29c-3p suppresses colon cancer cell invasion and migration through inhibition of PHLDB2. Biochem Biophys Res Commun 2017, 487(1):90-95.

40. Fleming NI, Jorissen RN, Mouradov D, Christie M, Sakthianandeswaren A, Palmieri M, Day F, Li S, Tsui C, Lipton L, Desai J, Jones IT, McLaughlin S, Ward RL, Hawkins NJ, Ruszkiewicz AR, Moore J, Zhu HJ, Mariadason JM, Burgess AW, Busam D, Zhao Q, Strausberg RL, Gibbs P, Sieber OM. : SMAD2, SMAD3 and SMAD4 mutations in colorectal cancer. Cancer Research 2012, 73(2):725-35. 


\section{Figure 1}

\section{General characteristics of circRNAs in CRC}

(A) Genomic features of circRNAs expressed in human CRC. Chromosomal distribution of the circRNAs. (B) Distribution of the back-spliced exons in circRNAs. (C) Distribution of the number of different types of circRNA transcripts from each circRNA host gene. (D) Distribution of the number of back-spliced exons in each circRNA.
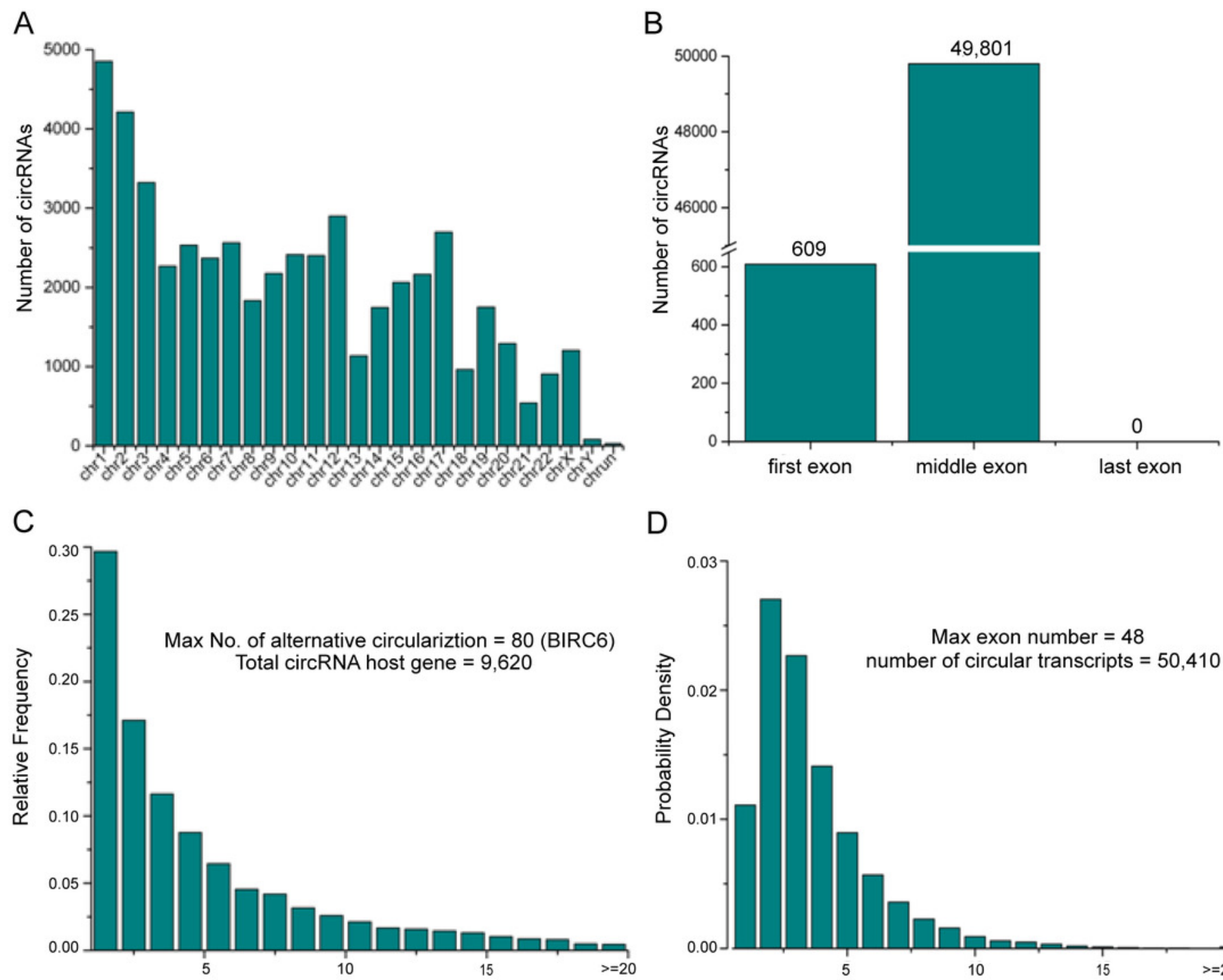

No. of circular transcripts in single refseq gene

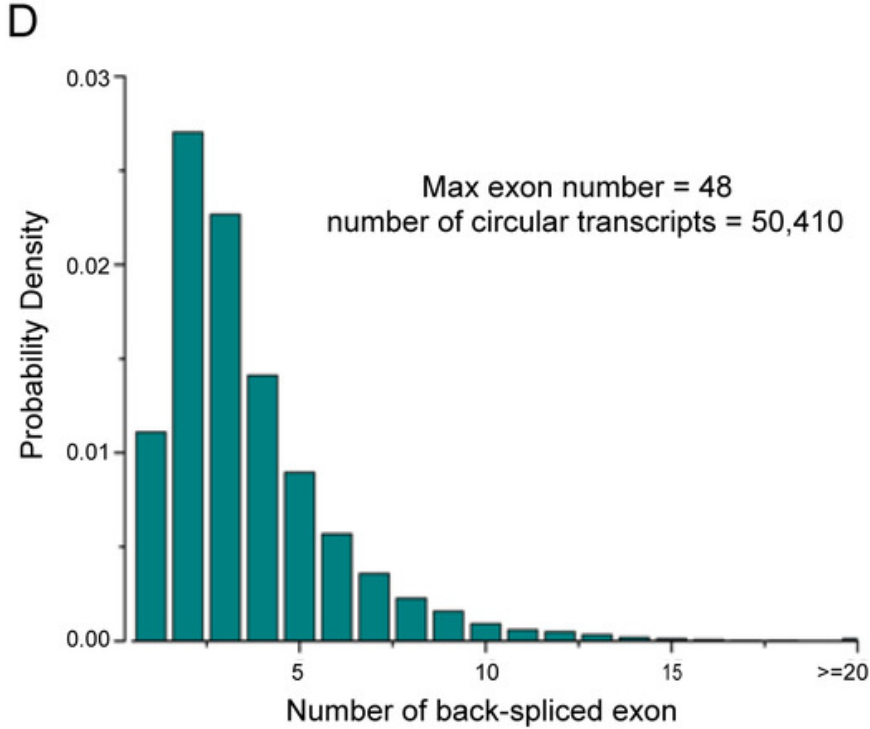




\section{Figure 2}

Differential expression of circRNAs in CRC tissues.

(A) Hierarchical clustering analysis of the circRNAs. CRC-D-A, CRC-D-B and CRC-D-C are adjacent normal tissue samples. The remaining three are cancer tissue samples. (B) Volcano plots are constructed using the fold-change values and q-values. The red dots in the figure represent statistically significant differentially expressed circRNAs. (C) Distribution of genomic regions that differentially expressed circRNAs derived from: exonic, intronic regions, etc. (D) Potentially encoded protein analysis of differentially expressed circRNAs. (E) GeneMANIA network of host genes of differentially expressed circRNAs.

A

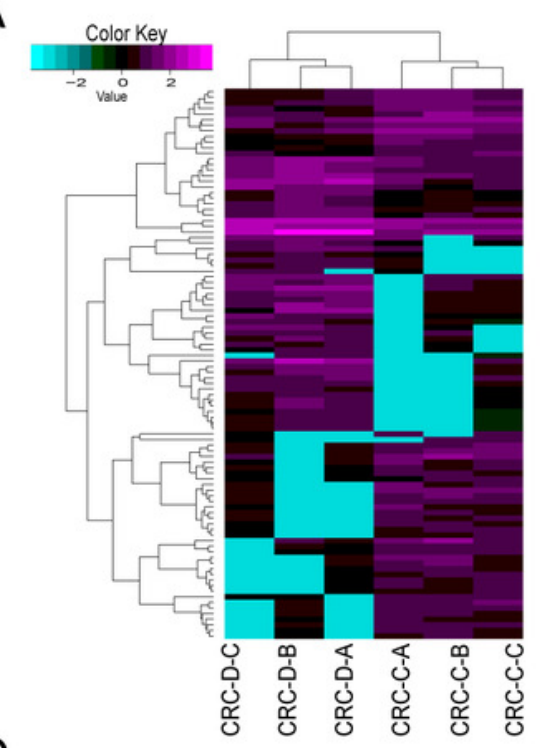

D

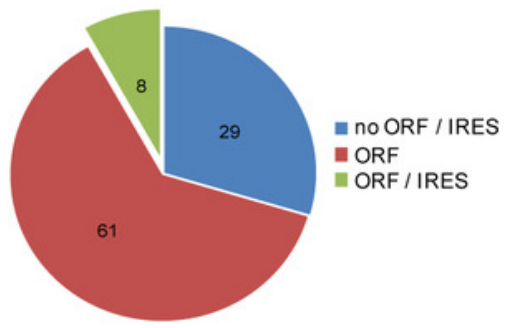

B

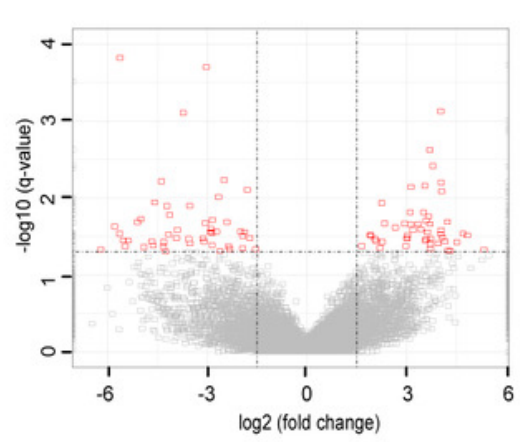

C

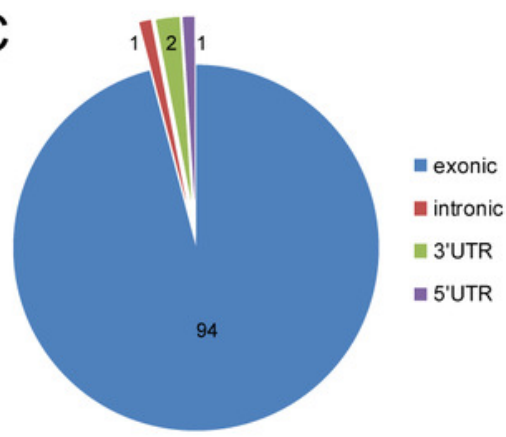

E

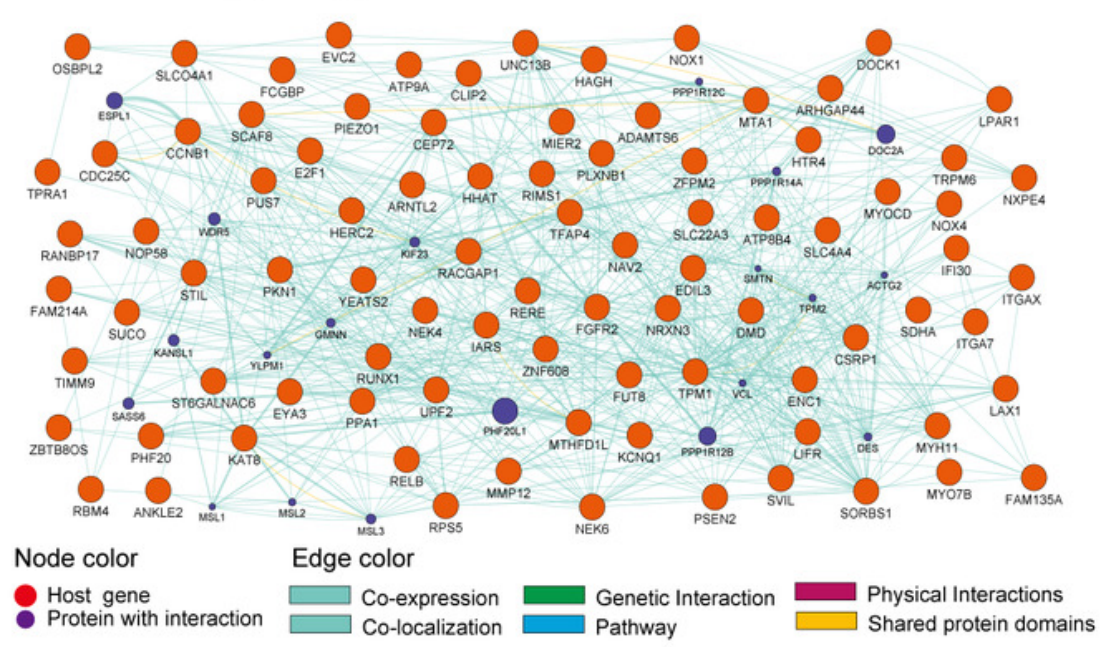




\section{Figure 3}

Differential expression analysis and interaction analysis of miRNAs and mRNAs

Volcano plots showing expression profile of pre-miRNAs (A) and mRNAs (B). (C) The intersection of the differentially expressed pre-miRNAs (DE_pmiRNA) and 1,666 pre-miRNAs. (D) The intersection of the differentially expressed mRNA (DE_mRNA) and 3707 miRNA target genes. (E) Comparison of data sizes before and after data processing. Purple indicates retained data and blue indicates discarded data. 
A

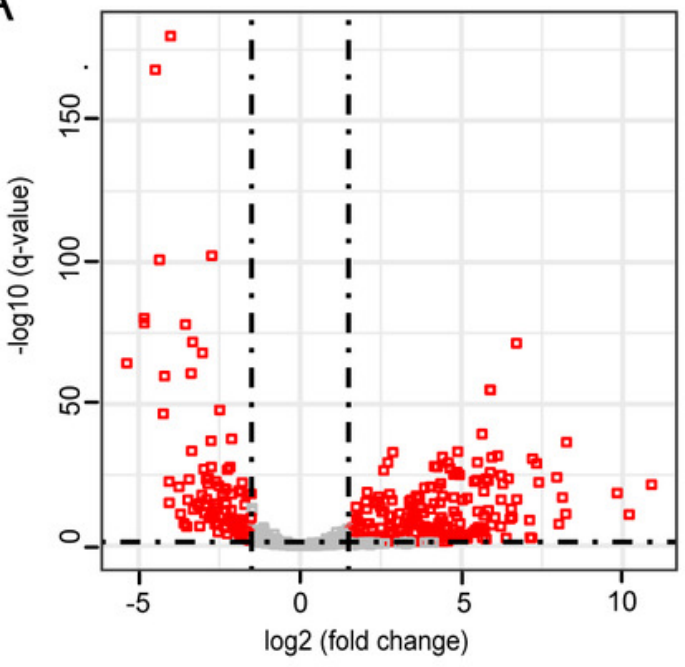

B

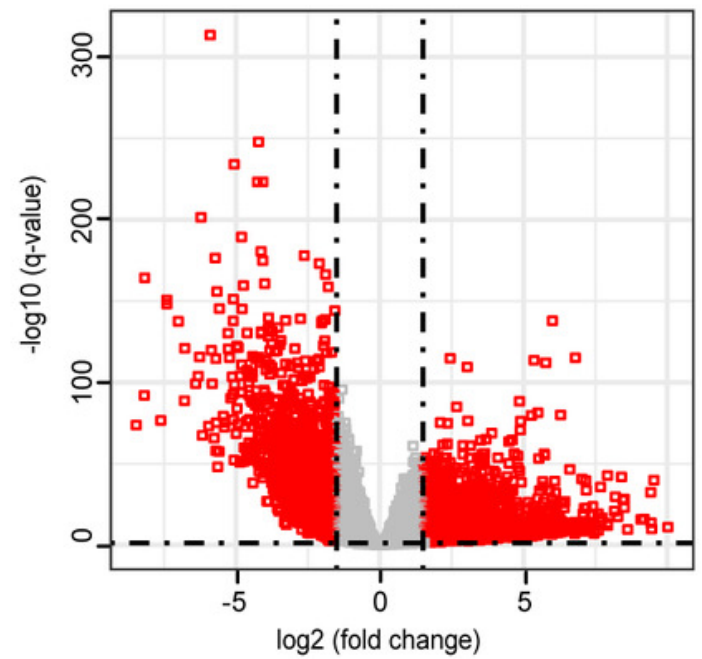

C

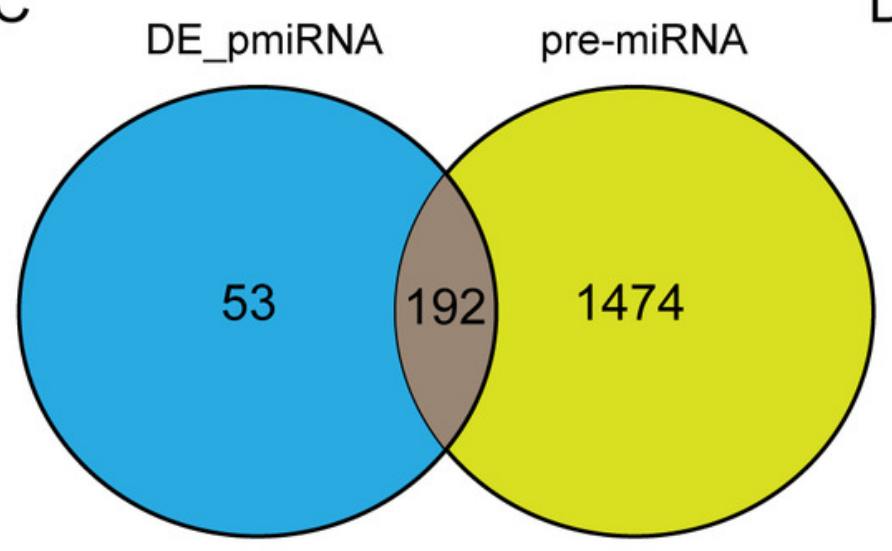

D

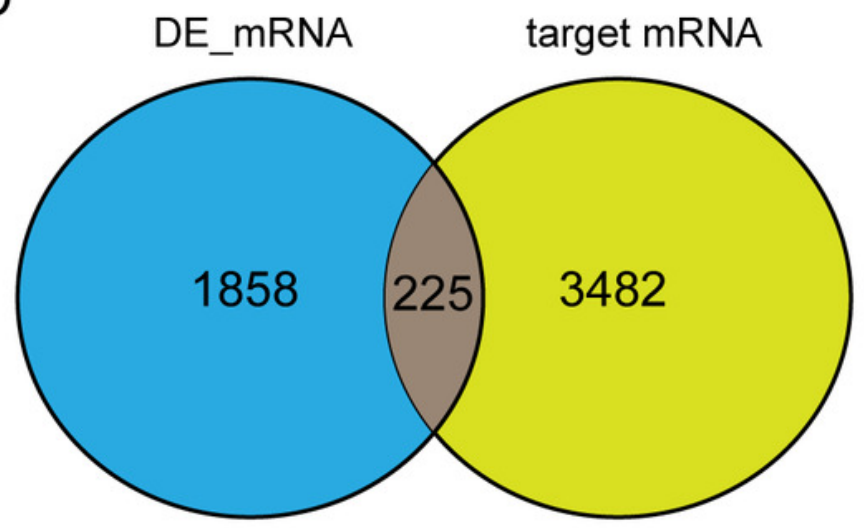

E

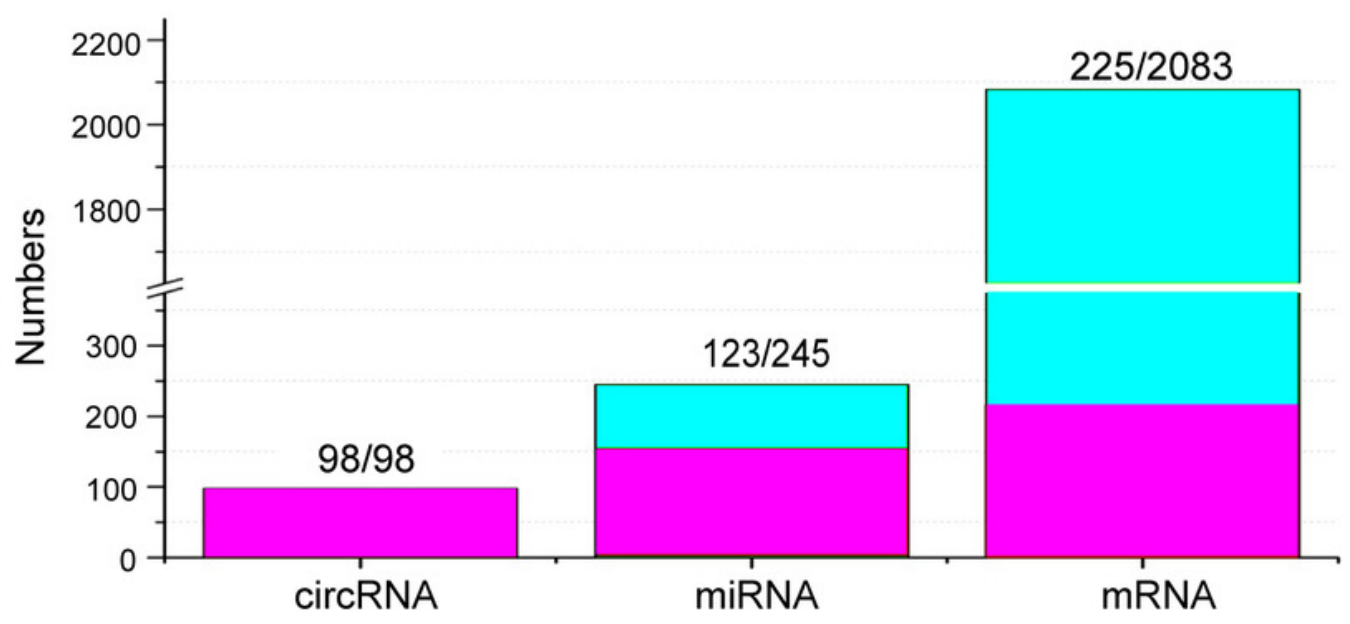




\section{Figure 4}

Information on six hub circRNAs

(A) The top three up-regulated and down-regulated circRNAs. The outer loop represents the exon that constitutes the circRNA, the innermost green ring represents the ORF, the middle red triangle represents the microRNA response element, and the blue cross point represents the RNA binding protein. (B) CircRNAs-miRNAs-mRNAs network. The red circle denotes the down-regulated circRNAs, the green circle denotes the up-regulated circRNAs, the blue inverted triangle denotes miRNAs, and the purple rectangle denotes the mRNAs. (C) Survival analysis of ceRNA - associated mRNA. (D) The KEGG pathway analysis of the top three circRNA pairs in the up-regulation and down-regulation circRNAs. 
A

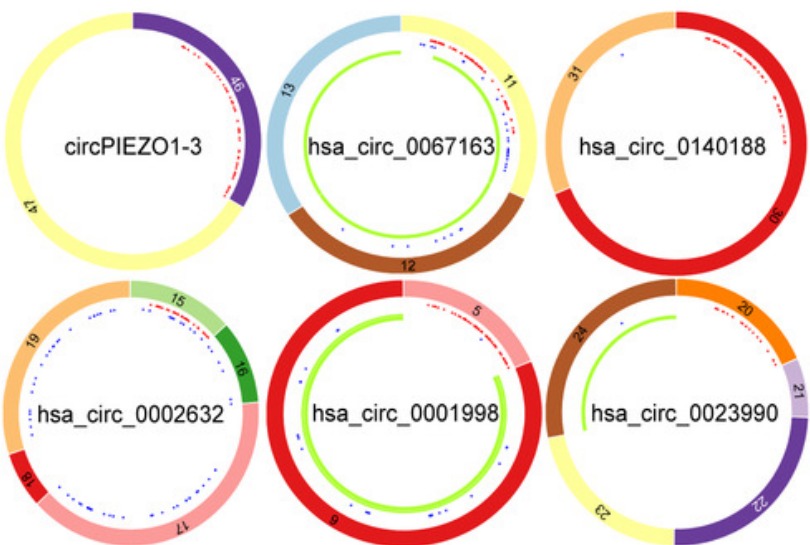

C
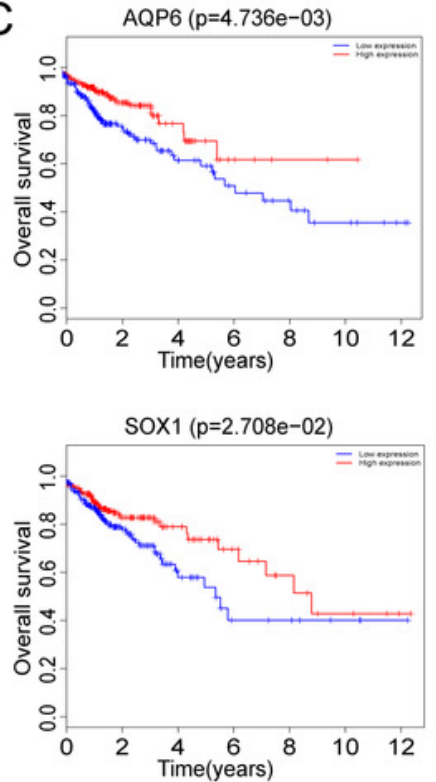
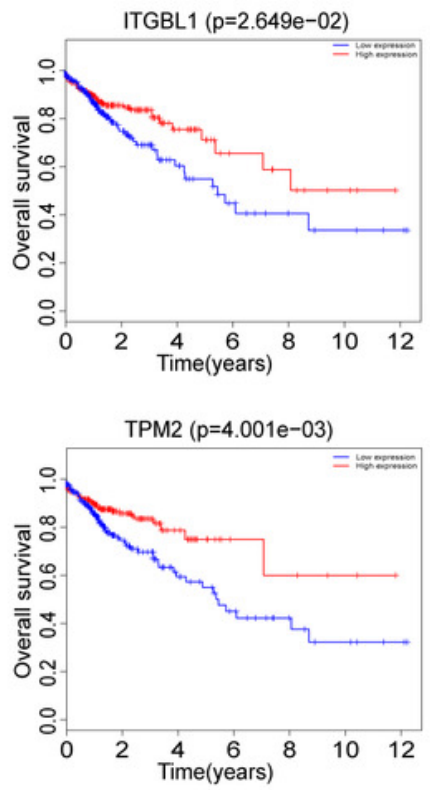

B
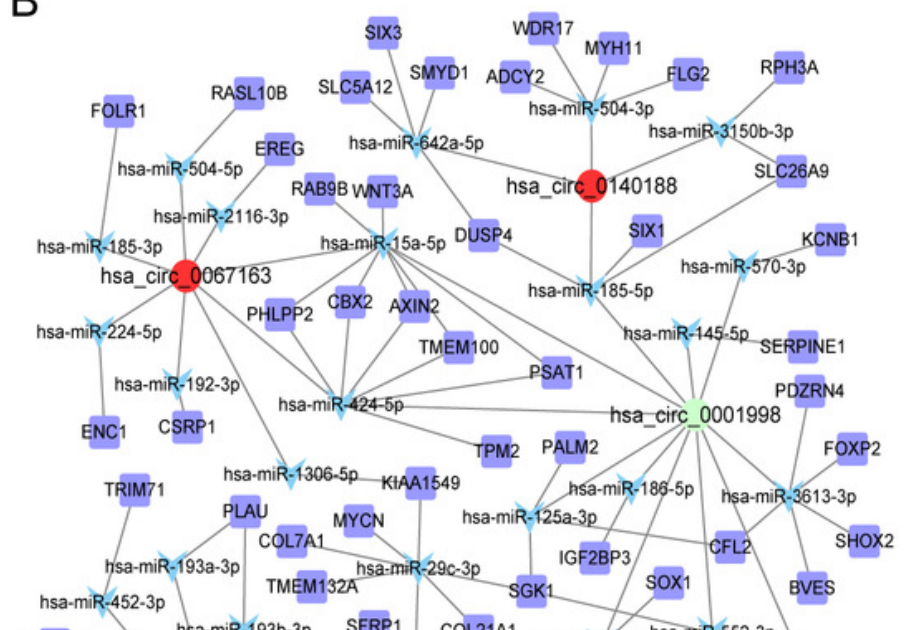

TMEFF2 hsa-miR-6720-5

nsa-miR-193b-3p SFRP1 COL21A1

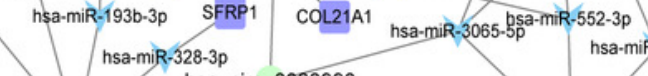
hsa-miR-3942-3p PLA2G2F
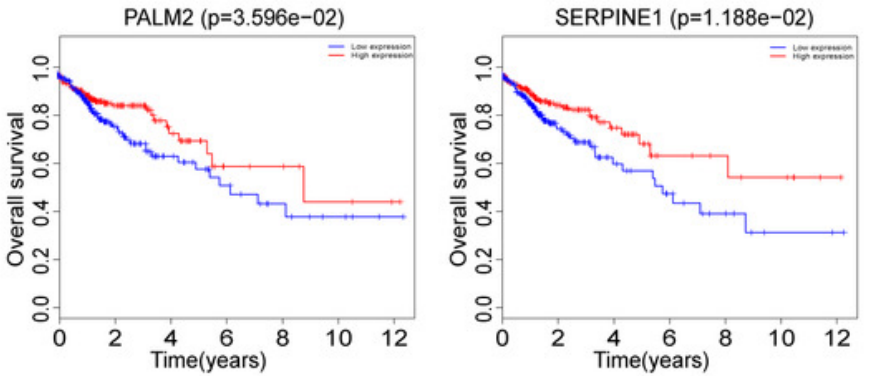

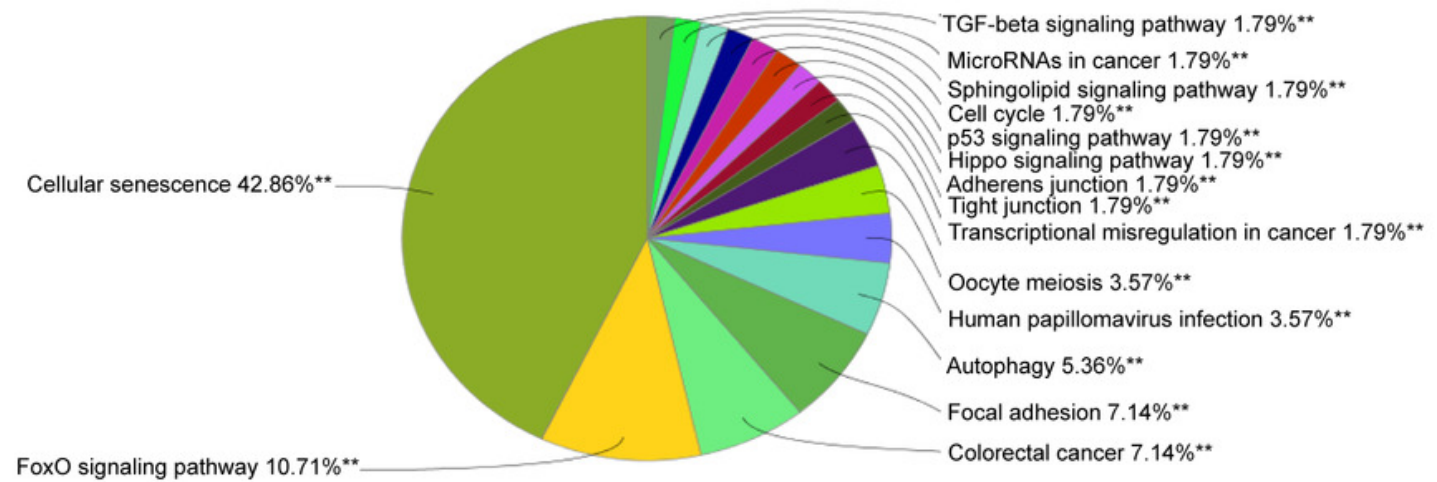

